\title{
Time-resolved imaging of the microbunching instability and energy spread at the Linac Coherent Light Source
}

\author{
D. Ratner, ${ }^{1, *}$ C. Behrens, ${ }^{1,2}$ Y. Ding, ${ }^{1}$ Z. Huang, ${ }^{1}$ A. Marinelli, ${ }^{1}$ T. Maxwell, ${ }^{1}$ and F. Zhou ${ }^{1}$ \\ ${ }^{1}$ SLAC National Accelerator Laboratory, 2575 Sand Hill Road, Menlo Park, California 94025, USA \\ ${ }^{2}$ Deutsches Elektronen-Synchrotron DESY, Notkestraße 85, 22607 Hamburg, Germany
}

(Received 17 December 2014; published 9 March 2015)

\begin{abstract}
The microbunching instability (MBI) is a well-known problem for high brightness electron beams and has been observed at accelerator facilities around the world. Free-electron lasers (FELs) are particularly susceptible to MBI, which can distort the longitudinal phase space and increase the beam's slice energy spread (SES). Past studies of MBI at the Linac Coherent Light Source (LCLS) relied on optical transition radiation to infer the existence of microbunching. With the development of the $\mathrm{x}$-band transverse deflecting cavity (XTCAV), we can for the first time directly image the longitudinal phase space at the end of the accelerator and complete a comprehensive study of MBI, revealing both detailed MBI behavior as well as insights into mitigation schemes. The fine time resolution of the XTCAV also provides the first LCLS measurements of the final SES, a critical parameter for many advanced FEL schemes. Detailed MBI and SES measurements can aid in understanding MBI mechanisms, benchmarking simulation codes, and designing future high-brightness accelerators.
\end{abstract}

DOI: 10.1103/PhysRevSTAB.18.030704

PACS numbers: 41.60.Cr, 29.27.-a, 29.20.Ej

\section{INTRODUCTION}

The linac microbunching instability (MBI) is a persistent challenge for high brightness electron beams, and freeelectron lasers (FELs) in particular. Microbunching has been studied extensively both theoretically [1-13] and experimentally [14-23]. In the standard model for the longitudinal space charge (LSC) flavor of MBI, density modulations in the beam produce LSC fields that modulate the beam energy, and a subsequent dispersive region generates a corresponding amplification in the density modulation. The high brightness beams, long accelerating sections, and strong dispersive regions at the Linac Coherent Light Source (LCLS) result in strong microbunching; the amplified density modulations can disrupt FEL operation both by producing intense radiation that disables diagnostics and by deforming the electron beam phase space, which directly degrades the FEL performance. With the tight parameters of future high-repetition rate machines [24], there is renewed interest in a detailed understanding of MBI.

Past studies of MBI at LCLS used coherent optical transition radiation (COTR) to investigate microbunching. With the x-band transverse deflecting cavity (XTCAV) [25] it is now possible to image the electron bunch's full longitudinal phase space at the end of the accelerator.

*dratner@slac.stanford.edu

Published by the American Physical Society under the terms of the Creative Commons Attribution 3.0 License. Further distribution of this work must maintain attribution to the author(s) and the published article's title, journal citation, and DOI.
We use a detailed analysis of the MBI behavior to identify the dominant sources of microbunching, and confirm the shot-noise based models of MBI [1,3,9]. Measuring microbunching as a function of laser heater amplitude [20,23] reveals the MBI gain response vs initial energy spread before compression. We can also use the final bunch compressor chicane to study methods for mitigating the instability.

A second advance due to the XTCAV is the ability to measure the final slice energy spread (SES) with time resolution near the FEL coherence length. Though typically small enough not to affect LCLS lasing, SES limits more advanced schemes such as high gain harmonic generation [26], harmonic lasing [27,28], longitudinal space charge amplifiers [29,30], and dispersive noise suppression [31,32]. Here we report LCLS's first measurements of SES of the accelerated beam, as well as SES dependence on the laser heater and MBI.

\section{MICROBUNCHING INSTABILITY}

\section{A. Microbunching metric}

In MBI, an initial density modulation on the electron beam produces space charge forces that modulate the energy along the bunch. When the beam passes through a dispersive region, the particles move longitudinally according to the energy modulation, enhancing the initial density modulation. The initial modulation can be driven intentionally from an external source [33], but at LCLS is assumed to develop from shot noise (see e.g., [10]).

To quantify the degree of microbunching, we calculate the bunching factor $|b(k)|$ of a measured current profile $I(z)$ from 


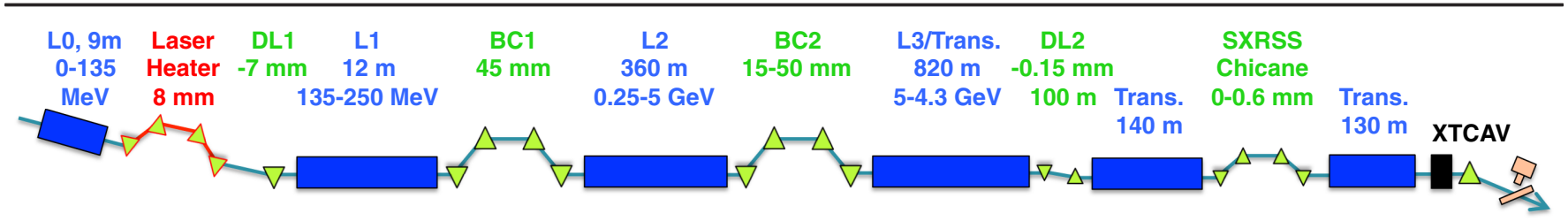

FIG. 1. Schematic of the LCLS accelerator showing the accelerating/transport (blue) and dispersive regions (green) that contribute to MBI. For dispersive regions, the $R_{56}$ is given in mm. Diagram includes the laser heater (in red), accelerating sections (L0-L3), transport sections (trans.), bunch compressors (BC1-BC2), doglegs (D1-D2), and the soft x-ray self-seeding chicane (SXRSS).

$$
b(k) \equiv \frac{1}{L} \int d z \Delta I(z) e^{-i k z},
$$

with longitudinal position $z$, wave number $k=2 \pi / \lambda$ at the point of measurement, bunch length $L$, and differential current $\Delta I(z) \equiv I(z) / I_{0}(z)-1$ defined as relative fluctuations around a smoothed "average" current $I_{0}(z)$ (see Appendix A). All measurements in this paper are made at the end of the accelerator at the XTCAV (Fig. 1), so unless otherwise noted, wavelengths in this paper refer to measured microbunching after bunch compression. The bunching factor from a single MBI stage increases by a gain factor (see e.g., [34])

$$
G(k) \approx \frac{I_{0} k}{C \gamma I_{A}}\left|R_{56} \int_{0}^{\ell} d s \frac{4 \pi Z(k / C, s)}{Z_{0}}\right| \exp \left(\frac{-k^{2} R_{56}^{2} \delta^{2}}{2}\right)
$$

with compression factor $C$, chicane longitudinal dispersion $R_{56}$, accelerator length $\ell$, electron energy $\gamma$, accelerator impedance $Z$, free space impedance $Z_{0}=377 \Omega$, Alfven current $I_{A}=17 \mathrm{kA}$, and relative SES before the chicane of $\delta$. In the low frequency regime, $\left(k \sigma_{r} / C \gamma \ll 1\right.$ with beam size $\sigma_{r}$ ), the impedance is approximately

$$
Z(k / C) \approx \frac{i Z_{0} k}{4 \pi \gamma^{2} C}\left[1+2 \ln \left(\gamma C / k \sigma_{r}\right)\right]
$$

Plugging into Eq. (2) we find that the bunching factor increases as $k^{2}$ until reaching a cutoff wave number, $k_{c}=1 /\left(R_{56} \delta\right)$, where the energy spread suppresses gain exponentially. To prevent MBI gain at the more damaging shorter wavelengths, a laser heater increases $\delta$ before the instability degrades the beam quality $[6,7,20,23]$.

\section{B. Laser heater}

Prior studies of both FEL performance [20] and gain length [35] gave indirect indications of the laser heater's effectiveness. Previous measurements of microbunching relied on COTR; when a microbunched beam passes through a thin metallic foil, the current modulations drive COTR at the wavelength of the modulation [10,14,15]. Though sensitive to small modulations, COTR is still an indirect measurement that provides limited information. In this paper we use the high resolution of the XTCAV to image the full longitudinal phase space of the beam. Figure 2 shows a few example images at different laser heater settings. Strong microbunching is evident even by eye, as is the reduction in microbunching as the laser heater amplitude increases. Figure 3 shows a projection of the current density when the laser heater is off; with modulations up to $50 \%$ of the average current, compared to order of $0.01 \%$ from shot noise, the MBI has essentially saturated.

We can now use the XTCAV to make a direct study of the laser heater's effect on MBI. Figure 4 shows measured
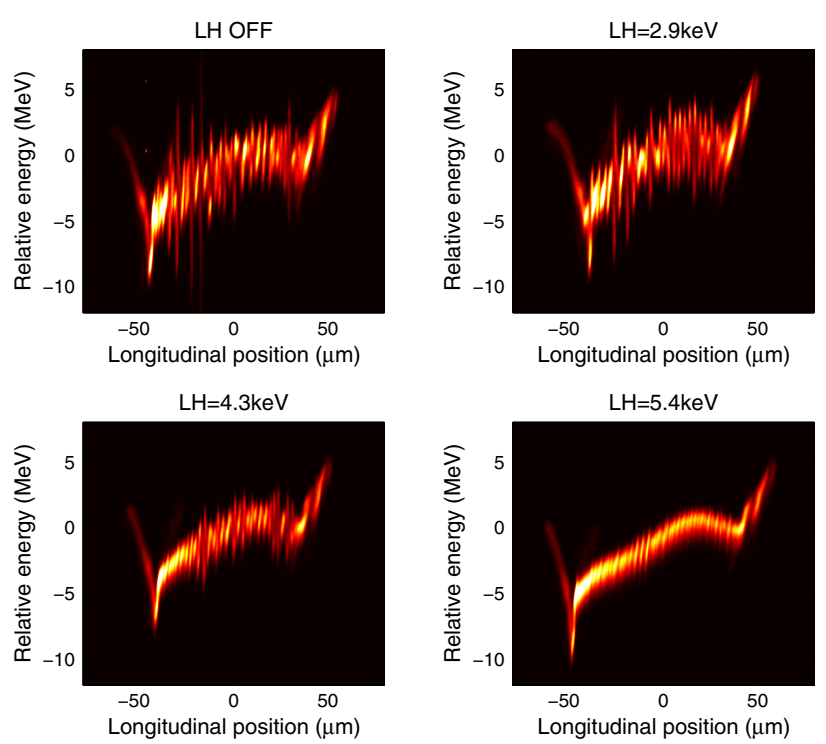

FIG. 2. Measured electron phase space with different rms energy spread induced by the laser heater (LH). Conditions are $0.5 \mathrm{kA}$ peak current, $44 \mathrm{MV}$ XTCAV voltage, and bunch head to the right.

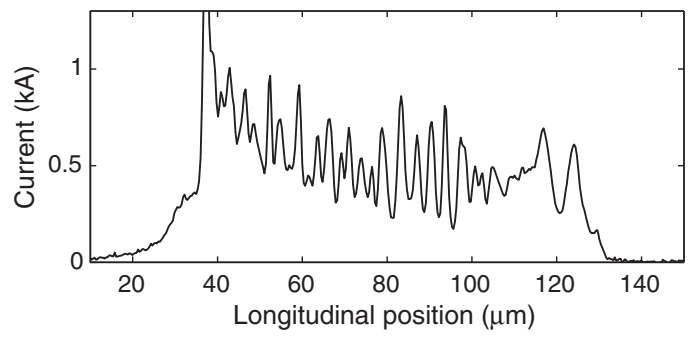

FIG. 3. Current taken from a projection of the upper left image in Fig. 2. Strong modulations show that MBI is nearly saturated without the laser heater. 

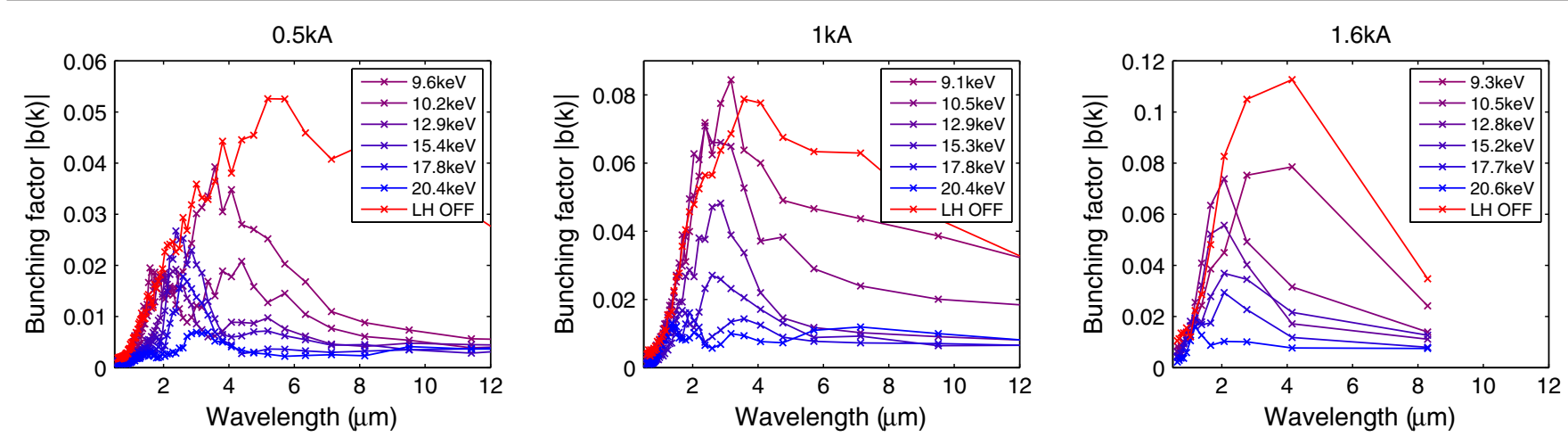

FIG. 4. Bunching factor as a function of energy spread induced by the laser heater for peak currents of $0.5 \mathrm{kA}$ (left), $1 \mathrm{kA}$ (center), and $1.6 \mathrm{kA}$ (right). Each curve is a 30 shot average with $20 \%$ error bars (not shown for clarity). Vertical scale changes depending on bunch length (and hence current) because of the short MBI coherence length. Note that laser heater conditions were different for data shown in Fig. 2. Experimental details in the Appendix.

bunching directly as a function of induced energy spread from the heater. The three peak current cases show similar gain behavior with peak bunching in the range of $1.5-4 \mu \mathrm{m}$. The XTCAV resolves bunching down to $1 \mu \mathrm{m}$, so the cutoff is not from diagnostic resolution. (See Appendix B and [25] for details.) We note that the peak microbunching shifts to shorter wavelengths as the heater amplitude increases, shown in Fig. 5(a); the larger initial energy spread suppresses MBI-induced heating, which leads to smaller $\delta$ further down the accelerator, and thus larger $k_{c}$. Equation (2) describes a single-stage of

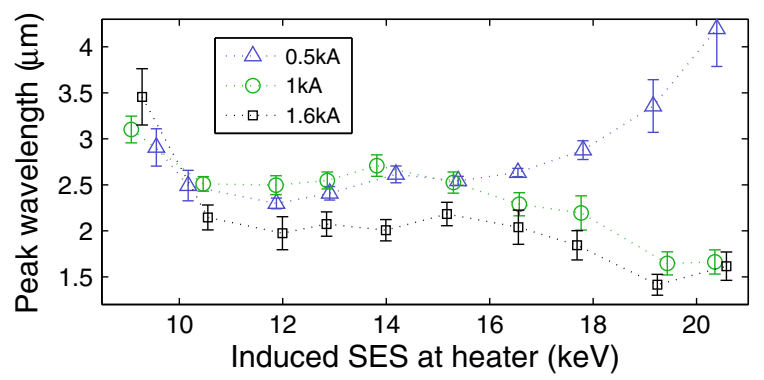

(a)

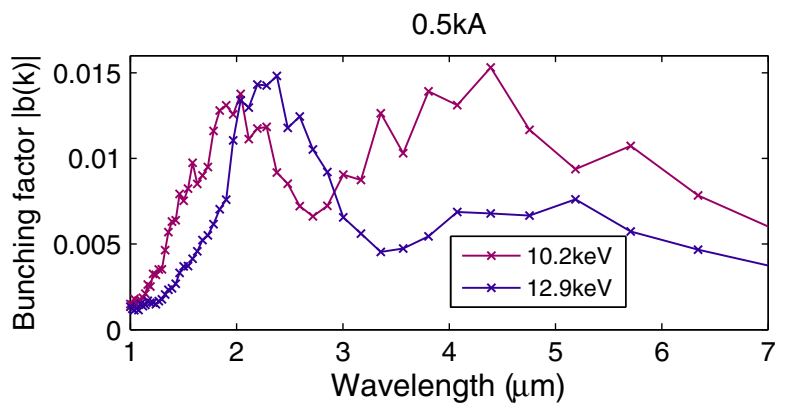

(b)

FIG. 5. (a) The peak bunching factor shifts to shorter wavelengths as the heater amplitude increases, but the behavior is more complicated at large amplitude. (b) A zoom of two curves from Fig. 4(a) highlights the complex behavior of the multi-stage MBI, such as a double peak in the bunching factor.
MBI, but with multiple stages, some with bunch compression, the detailed behavior is more complicated; e.g., with larger heating in Fig. 5(a) the peak shifts back to longer wavelengths in the $0.5 \mathrm{kA}$ case, and some curves from Fig. 4(a) show a notable double-humped distribution (Fig. 5(b)).

\section{BC2 dispersion}

The second bunch compressor $(\mathrm{BC} 2)$ is the last strong dispersive section, so it is expected that this chicane dominates the measured bunching factor. The similarity of the bunching factor curves for 0.5-1.6 kA, which differ only by $\mathrm{BC} 2$ compression, also suggests $\mathrm{BC} 2$ dominates bunching. The weak effect of the soft x-ray self-seeding chicane (Appendix C) confirms that downstream beam line elements have negligible impact on bunching. In normal operation $\mathrm{BC} 2 R_{56}=25 \mathrm{~mm}$, but we can also change the dispersive strength. Equation (2) predicts that larger $R_{56}$ increases gain, but also shifts the cutoff to longer wavelengths, where the impedance [Eq. (3)] is smaller. Moreover, it is known that intentionally adding a dispersive region ("phase mixing") can reduce the amplitude of MBI $[19,22,36,37]$. While past studies assumed the electron beam has no energy chirp in the phase mixing chicane, sample images in Fig. 6 show that phase mixing is also effective in a bunch compressor, and that larger $R_{56}$ can improve the final beam quality. From the bunching factor in Fig. 7, we see that larger $R_{56}$ suppresses bunching at the wavelengths that drive the largest energy modulations, and that operating $\mathrm{BC} 2$ at larger $R_{56}$ can reduce MBI effects. This result may also suggest that in some parameter regimes a second bunch compressor can even decrease the impact of MBI [36].

\section{Longitudinal coherence length}

The microbunching instability at LCLS is expected to start from shot noise $[10,15]$, and the XTCAV measurements support this model. When including compression, 

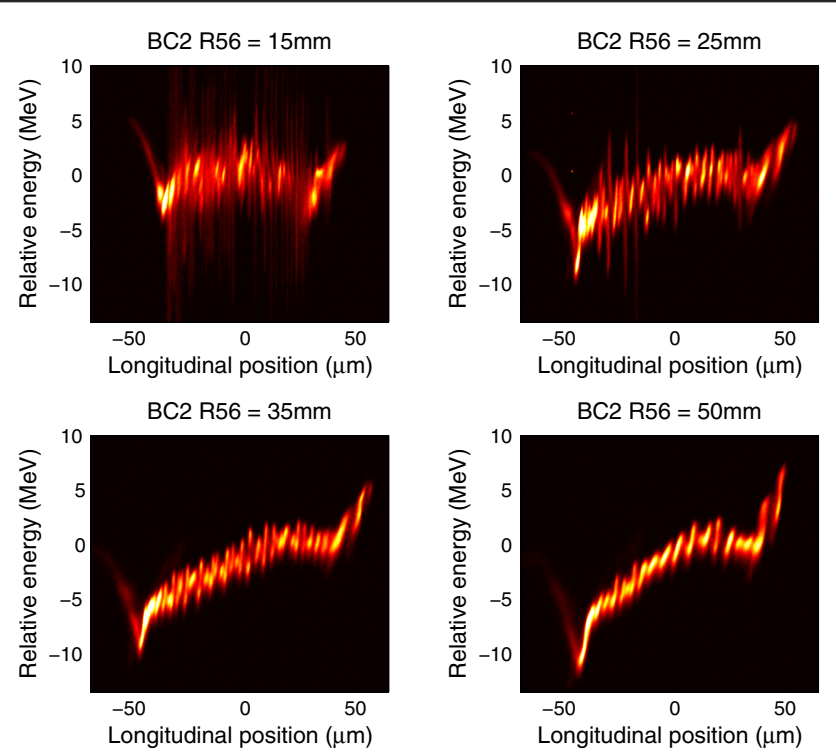

FIG. 6. Measured electron phase space imaged with the XTCAV for different values of BC2 $R_{56}$, but with the same total compression ( $0.5 \mathrm{kA}$ peak current). All images are with LH off, 44 MV XTCAV voltage, and bunch head to the right.

COTR data from prior experiments observed microbunching seeded at wavelengths from 0.5 to $50 \mu \mathrm{m}$. The XTCAV results in this paper correspond to seed wavelengths (before compression) ranging from 30 to $250 \mu \mathrm{m}$. The broad wavelength range is characteristic of shot noise, and is not consistent with a seed from the narrow bandwidth injector laser.

Second, because the XTCAV captures the electron beam's full temporal pulse structure shot-by-shot, it is possible to measure the coherence properties of the bunching. Figure 8(a) shows that while the average bunching factor curve is stable over time, the shot-by-shot bunching at a given $k$ fluctuates by $100 \%$. We can also find the MBI coherence length directly from the measured current profile by calculating the auto-correlation

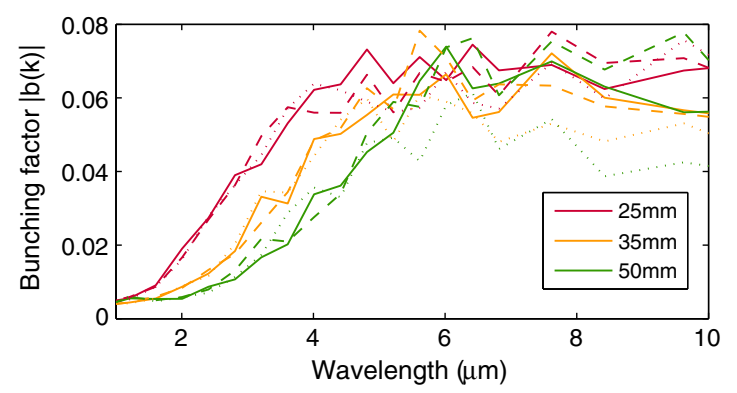

FIG. 7. Bunching factor for different settings of BC2, with $R_{56}$ ranging from $25 \mathrm{~mm}$ to $50 \mathrm{~mm}$. Chirp between BC1 and BC2 is changed to maintain the same total compression factor (peak current of $0.5 \mathrm{kA}$ ) for each case. Measured SES at the laser heater was $12 \mathrm{keV}$ (solid lines), $12.5 \mathrm{keV}$ (dashed lines), and $13 \mathrm{keV}$ (dotted lines).

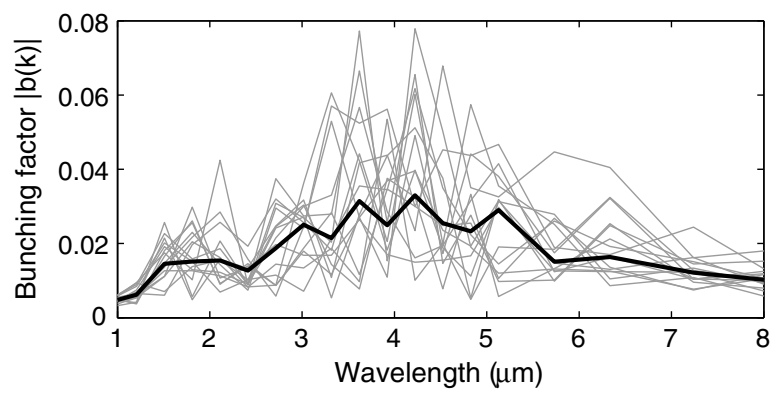

(a)

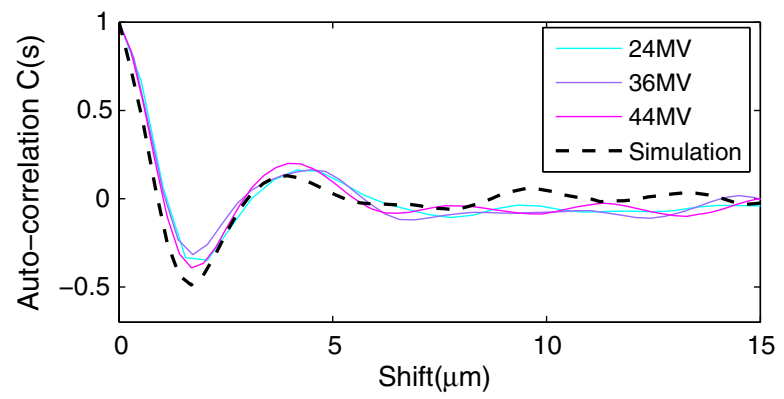

(b)

FIG. 8. (a) Single-shot (grey) and average (black) bunching factor with $0.5 \mathrm{kA}$ and induced energy spread of $10 \mathrm{keV}$ from the laser heater. Shot-to-shot fluctuations are consistent with a shotnoise seed. (b) Measured autocorrelation [Eq. (4)] compared to shot-noise simulation. The coherence length is independent of XTCAV voltage, showing the correlation is not resolution limited.

$$
C(s)=\frac{1}{C_{0}} \int d z \Delta I(z) \Delta I(z-s),
$$

with normalization $C_{0} \equiv \int d z \Delta I(z)^{2}$. Figure 8(b) shows the resulting correlation, averaged over 50 shots. As a check of the model, we use the measured spectral bunching factor to estimate a gain curve and apply it to a simulated shot-noise beam; Fig. 8(b) then compares the simulated auto-correlation to the measured correlation function. The shot-to-shot fluctuations and short MBI coherence length are both consistent with a shot-noise model.

\section{SLICE ENERGY SPREAD}

In the previous sections, we have seen that MBI can drive strong longitudinal density modulations (i.e., current spikes). These density modulations arise from local modulations of the beam energy (evident in Figs. 2 and 6), so in addition to the current spikes, MBI also tends to increase SES. While it was previously possible to measure the projected energy spread, strong wakefields throughout the accelerator result in a projected energy spread that is significantly larger than the SES. However, with the fine time resolution of the XTCAV, it is now possible to characterize the SES of the electron beam at the end of the accelerator. Moreover, because SES is essential to a 
number of advanced FEL schemes, such as high gain harmonic generation [26], harmonic lasing [27,28], and dispersive noise suppression [31,32], there is strong interest in measuring the slice characteristics of the beam.

Measuring SES requires lower XTCAV voltage compared to the MBI studies. XTCAV voltage is set high enough to resolve SES (near the FEL coherence length, $\sim 1 \mu \mathrm{m}$ at $4.3 \mathrm{GeV}$ ), but low enough so that heating by the Panofsky-Wenzel (PW) effect [38] is moderate and can be subtracted off in quadrature (see Appendix E). To avoid FEL-induced energy spread, all undulators are removed during measurements.

Energy spread suppresses MBI gain [Eq. (2)], so intentionally heating the beam slightly with a laser heater prevents the beam from overheating itself via MBI $[6,7,20,23]$. To determine conditions with minimum energy spread, Fig. 9 shows rms SES in the core of the beam as a function of the laser heater amplitude. At low heater amplitudes, MBI saturates and ruins the beam phase space. At high heater amplitudes, the heater suppresses MBI effectively, but the heater itself increases SES [20,23,35]. Results with LCLS parameters setup for soft x-rays are given in Table I. Measurements at higher electron energies

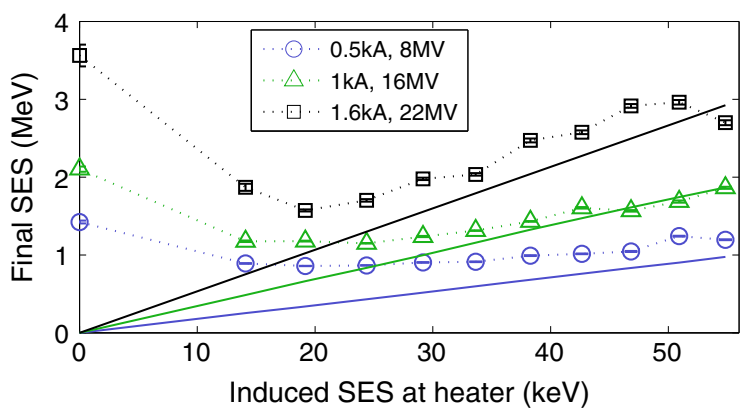

(a)

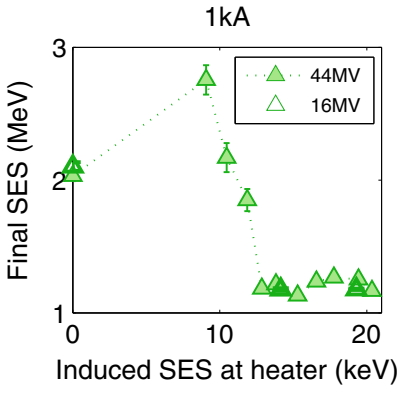

(b)

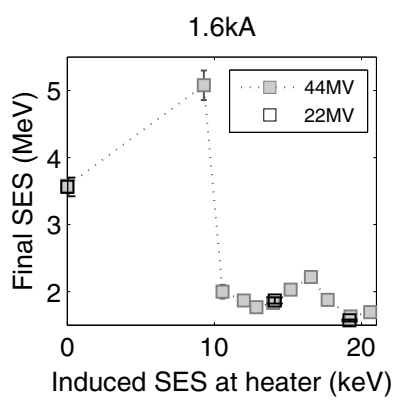

(c)
FIG. 9. (a) Final rms SES as a function of induced SES from the laser heater. Solid lines show the expected energy spread from phase space compression, assuming an initial current of $28 \mathrm{~A}$. Below, finer scans at lower heater settings for peak currents of $1 \mathrm{kA}$ (filled triangles, (b) and $1.6 \mathrm{kA}$ (filled squares, (c) overlaid with corresponding data from (a). In both cases a low level of heating actually increases energy spread compared to heater off. We ignore the beam's initial SES, which is negligible compared to that induced by the laser heater and MBI. In all plots "MV" refers to XTCAV voltage.
TABLE I. Measured SES (rms) at $4.3 \mathrm{GeV}, 180 \mathrm{pC}$ with nominal laser heater setting (20 keV induced spread). Peak current is measured in the center of the beam.

\begin{tabular}{lccc}
\hline \hline Peak current (kA) & 0.5 & 1 & 1.6 \\
SES $(\mathrm{MeV})$ & 0.9 & 1.2 & 1.6 \\
\hline \hline
\end{tabular}

are challenging, but MBI is weaker and SES may actually be smaller.

It is interesting to compare the measured SES to expectations for an ideal beam. In the absence of MBI, the final SES is simply the product of the compression factor and the initial SES (due to the laser heater) measured at the beginning of the linac. To calculate the compression, we measure both the initial and final peak currents. Previous measurements have found an initial peak current around 30 A for our parameter set, and from the regime with maximum heater strength we find a best fit of $28 \mathrm{~A}$. We use the XTCAV images to measure the final peak current in the core of the beam. The resulting predicted SES in the absence of MBI is shown as the solid lines in Fig. 9(a). Assuming contributions add in quadrature, we find that even at the point of minimum SES, MBI still contributes a larger portion of SES than comes from the laser heater. Thus, it is clear that suppressing MBI with a mechanism that does not heat the beam (e.g., using a transverse cavity [39]) would allow for smaller final SES and could benefit future FELs.

The XTCAV only gives the SES at the end of the accelerator, but we can use the measured microbunching to infer SES at BC2. From initial conditions of $30 \mathrm{~A}$ and $3 \mathrm{keV}$ SES [10], bunch compression would produce just $25 \mathrm{keV}$ SES at BC2. However, with the laser heater off, Fig. 4 matches a wavelength cutoff at $3 \mu \mathrm{m}$, which implies actual SES at BC2 of $130 \mathrm{keV}$. So while behavior in L3 dominates the final SES, it appears that MBI increases the energy spread substantially even before $\mathrm{BC} 2$.

Figure 9 shows the general trend that increasing heater strength first reduces SES (by suppressing MBI), and then eventually increases SES (due to compression of the initial SES). However, at low intensities, we observe more complex behavior, just as with microbunching. Figures 9(b)-(c) shows the SES analysis applied to the MBI data of Figs. 4(b)-(c). We see that with the heater just barely on, SES is actually higher than with the heater off, which is consistent with the stronger impedance associated with the corresponding shift to shorter wavelength microbunching evident in Figs. 4(b)-(c).

The XTCAV images also show SES as a function of position along the beam, as seen in Fig. 10. At optimal laser heating, the core of the bunch has uniform SES, but with the laser heater off, the SES varies with longitudinal position. As expected, wakefields cause a large increase in the energy spread at the ends of the bunch even when the current spikes are moderate. 


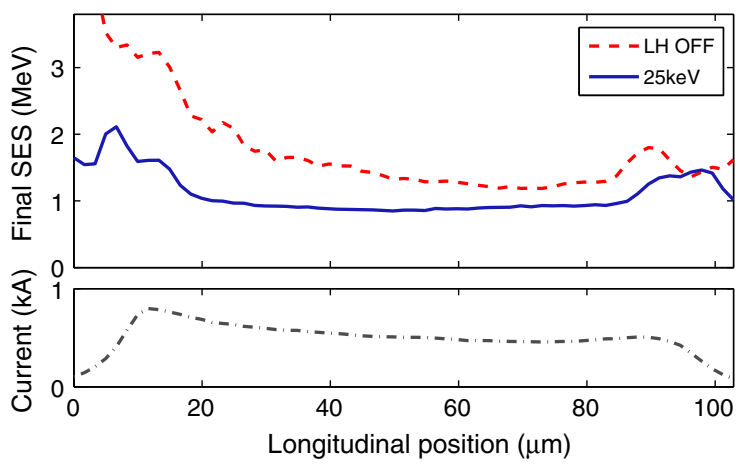

FIG. 10. A 30-shot average of SES as a function of position along the bunch for the laser heater off (dashed red line) and with induced energy spread at the heater of $25 \mathrm{keV}$ (solid blue line). Bottom plot shows current as a function of position along the bunch, with approximately $0.5 \mathrm{kA}$ at the core.

\section{SUMMARY}

The XTCAV reveals unprecedented details in the longitudinal phase space of an XFEL electron beam. While OTR diagnostics are more sensitive to low levels of microbunching and can probe shorter wavelengths, the XTCAV allows more complete, direct measurements of MBI. The microbunching analysis indicates complicated gain behavior, as expected for multi-stage MBI, and the data is consistent with shot-noise models. These results will be used to benchmark simulations for future machines. We also use the XTCAV to probe the energy spread of a beam slice of length $\sim 1 \mu \mathrm{m}$. We find that MBI has a strong effect

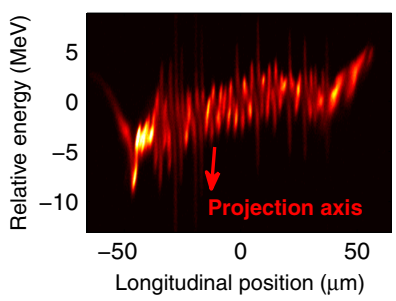

(a)

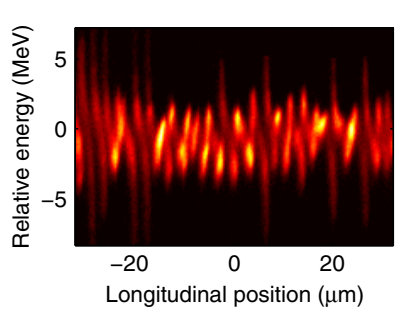

(e)

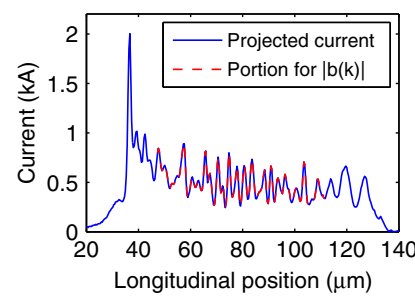

(b)

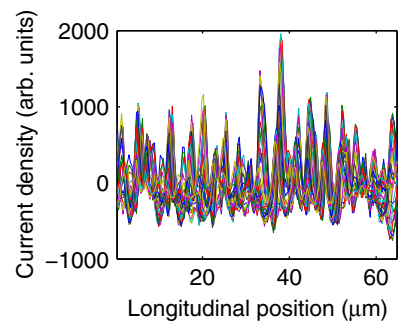

(f)

on the final beam conditions even with the laser heater at the nominal setting. The measured SES is an important input for future LCLS studies, including noise suppression and harmonic lasing. Finally, we observe that microbunching can be reduced by increasing the $R_{56}$ amplitude of the second bunch compressor, and future studies will investigate LCLS operation at these settings.

\section{ACKNOWLEDGMENTS}

Work is supported by Department of Energy Contract No. DE-AC02-76SF00515.

\section{APPENDIX A: MBI ANALYSIS METHODS}

To quantify the degree of microbunching, we developed two analysis metrics and cross-checked them to ensure the results were consistent. First, we calculate the standard bunching factor from

$$
b(k) \equiv \frac{1}{L} \int d z \Delta I(z) e^{i k z} .
$$

To find the current fluctuations, $\Delta I(z)=I(z) / I_{0}(z)-1$, we first need to find the current, $I(z)$, from a projection of the XTCAV image. A small, leaked horizontal dispersion causes a slight shearing of the electron phase space images, so we take a projection at the angle that maximizes the fluctuations $\Delta I(z)$. For the data set of Fig. 4, the projection was taken at an angle of $0.1 \mathrm{rad}$ (Fig. 11(a)). The exact tilt changes depending on the optics setup, but $0.1 \mathrm{rad}$ is

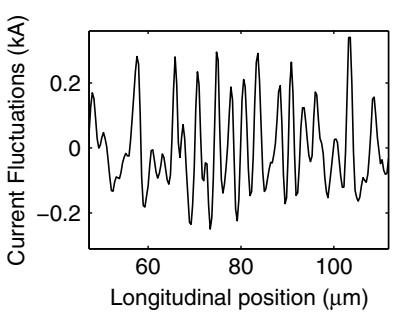

(c)

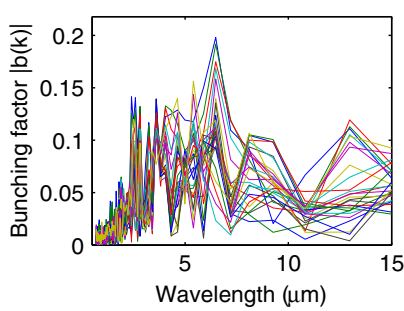

(g)

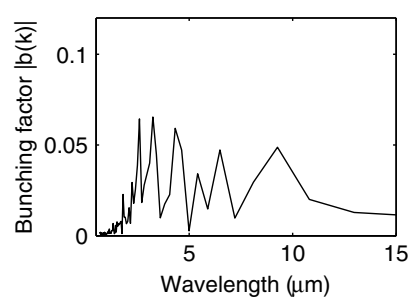

(d)

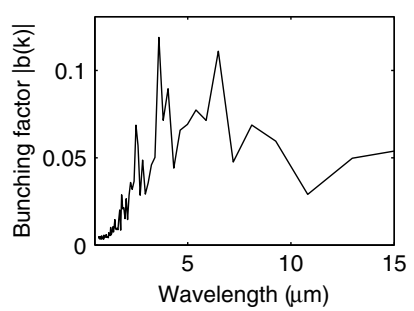

(h)

FIG. 11. Example of microbunching measurement for an image with $0.5 \mathrm{kA}$ peak current, laser heater off, and $44 \mathrm{MV}$ XTCAV voltage for both the normal (a-d) and modified (e-h) metrics. (a) Electron phase space imaged with the XTCAV. (b) Projected electron density at $0.1 \mathrm{rad}$, with only the center of the beam used for bunching factor calculations. (c) We remove the bunch curvature by fitting a second order polynomial to the central portion of the bunch current. (d) We calculate the bunching factor from the current fluctuations for a single shot. (e) For the modified method, we start by removing the energy and current curvature in the beam by fitting a fourth order polynomial. (f) We produce current fluctuations for each individual lineout of energy phase space and (g) calculate the bunching factor for each lineout. (h) Finally we average $|c(k)|^{2}$ to find the final bunching factor of a single shot. 
consistent with the ratio of vertical and horizontal dispersions inferred from synchronous beam position monitor data taken during the experiment. From the projection, we select only the core of the bunch (dotted red line, Fig. 11(b)) to avoid the wakefield spikes at the head and tail. To find the average current, $I_{0}(z)$, we fit a second order polynomial to $I(z)$ on a shot-by-shot basis to remove remaining effects from wakefield curvature. (Using the full current, $I(z)$, would emphasize the bunch form factor, rather than MBI effects.) The result for a single shot is given in Fig. 11(c). Finally, for each shot we calculate the bunching factor squared, $|b(k)|^{2}$, and average over 30 shots to produce the results in Fig. 4, presented as the amplitude $|b(k)|$.

To avoid the need for finding the correct projection axis empirically, and to check the results of the bunching measurements, we developed an alternative metric defined as

$$
|c(k)| \equiv \int d p f(p)\left|\frac{1}{L} \int d z \Delta I_{s}(p, z) e^{i k z}\right|,
$$

with electron energy $p$, normalized energy distribution $f(p)$, and current slice at a single energy $I_{s}(p, z)$. The energy slice current fluctuation, $\Delta I_{s}(p, z)$ is defined equivalently to $\Delta I(z)$. This approach, illustrated in Fig. 11(e)-(h) and which we will call the "modified" bunching factor, captures correlations in energy space that the standard bunching factor misses; for example, a pure sinusoidal energy modulation with no density modulation would have $|b(k)|=0$, but would have nonzero $|c(k)|$. However, the modified approach is not a standard metric, and it also has worse signal to noise ratio. Both methods give qualitatively similar results (Fig. 12), so we use the standard bunching factor in the main results of the paper.

Note that from Sec. II D, we find that the MBI coherence length is shorter than the bunch length, which implies that $|b(k)|$ will never reach unity even at saturation, and indeed $|b(k)|$ at saturation will depend on both the wavelength and bunch length. As a result, the measured bunching factor

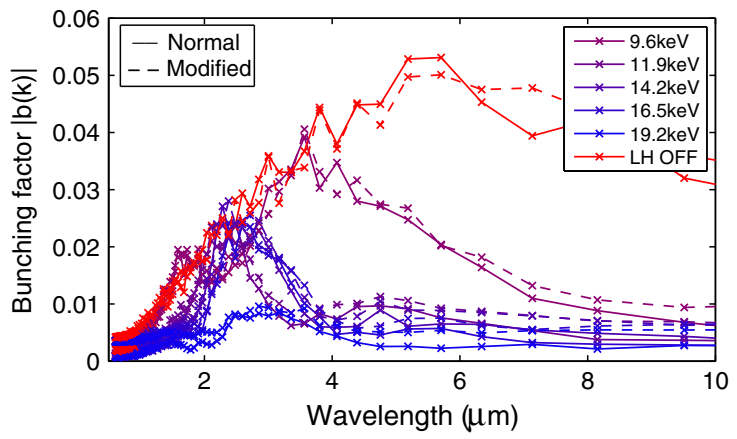

FIG. 12. A comparison of the bunching results at $0.5 \mathrm{kA}$ using normal bunching factor (solid lines) and modified bunching factor (dashed lines). levels are expected to be higher at high currents (when the bunch is shorter) and at long wavelengths (where there are fewer coherence lengths in the bunch).

\section{APPENDIX B: MBI RESOLUTION LIMIT}

The microbunching analysis shows little bunching below $1.5 \mu \mathrm{m}$ wavelength, so we would like to establish that this cutoff is due to MBI dynamics rather than XTCAV resolution. Figure 13(a) shows that the camera has pixel resolution of approximately $0.25 \mu \mathrm{m}$ (in principle allowing observation of microbunching at $0.5 \mu \mathrm{m}$ ). However, even with sufficient pixel resolution, it is possible that the electron optics smear out short wavelength bunching. The longitudinal resolution of a transverse horizontal deflector setup is given by

$$
\sigma_{z}=\frac{E_{e}}{k_{\mathrm{rf}} e V_{\mathrm{rf}}} \sqrt{\frac{\epsilon_{x}}{\gamma \beta_{x}}}
$$

where $E_{e}$ is the beam energy, $k_{\mathrm{rf}}$ is the deflector $\mathrm{rf}$ wavenumber, $V_{\mathrm{rf}}$ is the deflecting voltage, $\gamma$ is the relativistic factor, and $\beta_{x}$ and $\epsilon_{x}$ are the beta function and normalized emittance at the deflector, respectively. For the LCLS operating at $4 \mathrm{GeV}$ and $V_{\mathrm{rf}}=44 \mathrm{MV}$, we expect $\sigma_{z}<0.5 \mu \mathrm{m}$, consistent with reported measurements [25]. The resolution scales inversely with the XTCAV voltage, so we repeat the measurement with a range of voltages. Figure 13(b)-(c) shows the microbunching curve is unchanged across a factor of two change in voltage, confirming that the measurement is not resolution limited.

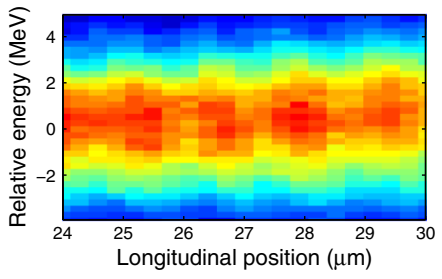

(a) (b)

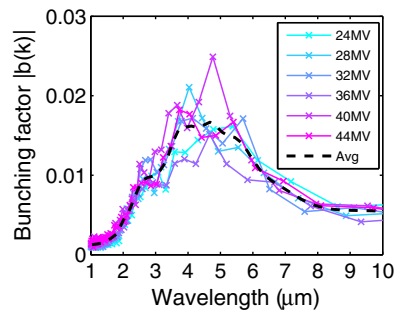

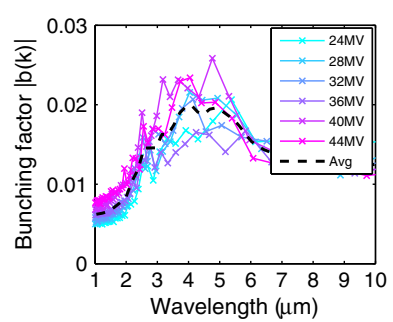

(c)
FIG. 13. (a) Electron beam image at $4.3 \mathrm{GeV}$ and XTCAV voltage set to $44 \mathrm{MV}$ showing camera pixel resolution of approximately $0.25 \mu \mathrm{m}$. (b) Standard bunching factor vs XTCAV voltage shows no evidence of resolution limits. (c) The modified bunching factor shows slightly higher short wavelength bunching with higher XTCAV voltage, but this may be due to the higher noise threshold at that setting. 
While the XTCAV provides the first images of the fully accelerated longitudinal phase space, the image analysis is not as sensitive as COTR to low levels of microbunching. With the laser heater generating more than $50 \mathrm{keV}$ SES before compression, microbunching is barely visible in the XTCAV image, but more sensitive COTR measurements show evidence of microbunching even with the laser heater producing $100 \mathrm{keV}$ SES [20].

\section{APPENDIX C: EFFECT OF SOFT X-RAY SELF-SEEDING CHICANE}

Studies indicate that $\mathrm{BC} 2$ determines the microbunching cutoff, which is not surprising; even though there is strong energy modulation after $\mathrm{BC} 2$, the only downstream dispersive region following the linac section L3 is the DL2 dogleg, which has just $-0.15 \mathrm{~mm} R_{56}$, and the equivalent $R_{56}$ of the entire L3 accelerating segment is less than $10 \mu \mathrm{m}$. We can use Eq. (2) to estimate the wavelength cutoff from DL2; for the worst case of $3.5 \mathrm{MeV}$ slice energy spread (from Fig. 9 with $1.6 \mathrm{kA}$ peak current and laser heater off), the cutoff would be less than $1 \mu \mathrm{m}$, so we do not expect DL2 to influence $k_{c}$. In addition, we observe experimentally that the wavelength cutoff is similar for the cases of $0.5-1.5 \mathrm{kA}$ (Fig. 4). The beam conditions are similar for all three peak currents until BC2, but differ by a factor of $>2$ in energy spread by DL2, which again

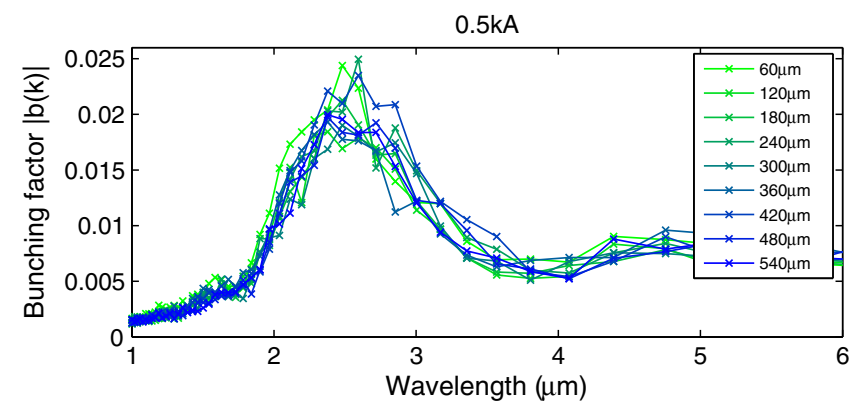

(a)

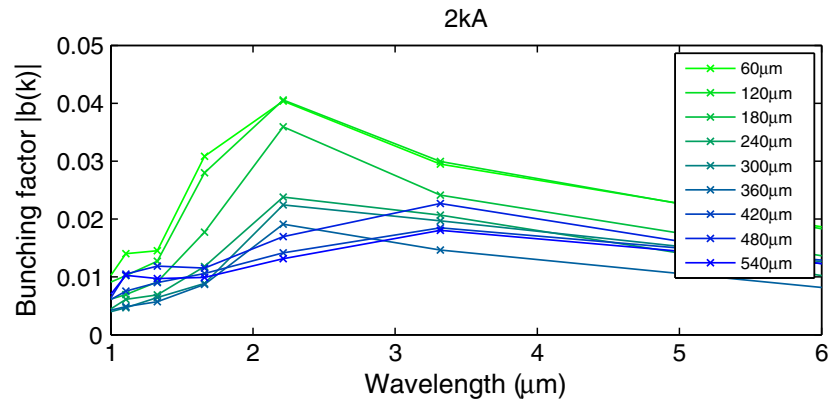

(b)

FIG. 14. Bunching factor vs SXRSS chicane strength for peak current and initial energy spread of (a) $0.5 \mathrm{kA}$ and (b) $2 \mathrm{kA}$. The chicane has negligible effect at $0.5 \mathrm{kA}$, and weak effect at $2 \mathrm{kA}$. Data taken with energy spread of $15 \mathrm{keV}$ induced by the laser heater. suggests that $\mathrm{BC} 2$ determines the measured microbunching cutoff.

As an experimental check that DL2 does not affect bunching, we modify the dispersion at the end of the accelerator. It is difficult to change the dispersion of DL2 directly, so instead we use a chicane installed for the soft xray self-seeding (SXRSS) project [40], approximately $100 \mathrm{~m}$ upstream of the XTCAV. The SXRSS chicane can scan from $0 \mathrm{~mm}$ up to $0.54 \mathrm{~mm} R_{56}$, almost a factor of 4 stronger than the nominal DL2 setting. Figure 14 shows that the chicane has no effect on the $0.5 \mathrm{kA}$ beam, and only a small effect on the $2 \mathrm{kA}$ beam. Using Eq. (2) and the observed microbunching dependence on $R_{56}$ in Fig. 14(b), we estimate SES of $3 \mathrm{MeV}$ at the SXRSS chicane. Given the uncertainty of the fit to the SXRSS chicane data, this is reasonable agreement with the direct XTCAV measurement of $2 \mathrm{MeV}$ under similar conditions in Fig. 9(c).

\section{APPENDIX D: RESOLUTION OF SLICE ENERGY SPREAD}

The energy spread measurements use the same XTCAV and diagnostics as for the MBI measurements, but the two studies employ different diagnostic parameters. While MBI requires maximum XTCAV voltage to resolve small longitudinal features, SES requires only sufficient streaking amplitude to differentiate slice from projected characteristics; excessive XTCAV voltage is counterproductive due to the Panofsky-Wenzel effect, described in Appendix E. Figure 15 shows a typical image for the case of a $0.5 \mathrm{kA}$ beam with a $30 \mathrm{keV}$ energy spread induced at the laser heater. The variation in the centroid of neighboring pixels is $0.25 \mathrm{MeV}$, more than a factor of 3 smaller than the measured energy spread of $0.91 \mathrm{MeV}$, so we conclude that we are resolving the slice, not projected, energy spread. By measuring the same SES at two different XTCAV voltages we can confirm that wake-field and RF-induced variations in correlated energy along the beam are not biasing the SES measurement (Fig. 9(b)).

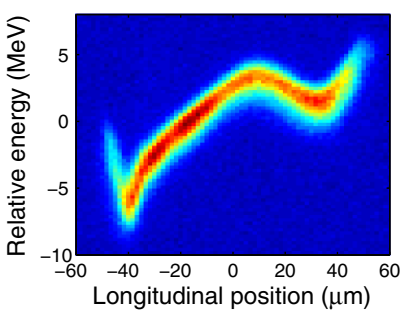

(a)

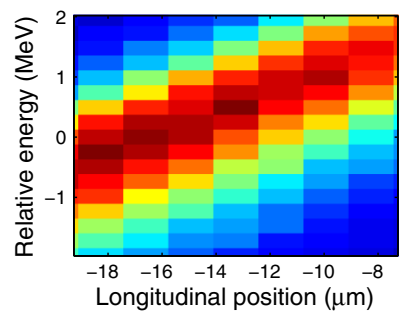

(b)
FIG. 15 . (a) Image of a $0.5 \mathrm{kA}$ beam, $30 \mathrm{keV}$ induced energy spread from the heater, taken with the XTCAV voltage at $8 \mathrm{MV}$. (b) Zoom of the same image shows the variation in the central energy of neighboring pixels $(0.25 \mathrm{MeV})$ is smaller than the measured SES (0.91 MeV). 
Note that the argument above only applies to correlated energy spreads at long length scales; there may well be short-distance correlations from MBI that cannot be resolved. While the FEL performance is determined by energy spread across the SASE coherence length (on the $\mu \mathrm{m}$ scale at soft $\mathrm{x}$-rays), lasing at hard $\mathrm{x}$-ray wavelengths as well as schemes such as optical klystron enhancement and noise suppression are sensitive to length scales that are too short to be resolved by the XTCAV $[31,41]$.

\section{APPENDIX E: ENERGY SPREAD, PANOFSKY-WENZEL EFFECT}

With the XTCAV turned to its maximum voltage, it is easy to resolve slice characteristics, as seen in the MBI measurements. However, the XTCAV itself affects the energy spread due to the Panofsky-Wenzel (PW) effect,

$$
\delta_{\mathrm{PW}}=e V k_{\mathrm{rf}} \sigma_{x},
$$

with electric charge $e$, and XTCAV parameters of voltage $V$, wave number $k_{\mathrm{rf}}$, and rms beam size $\sigma_{x}$. Assuming a Gaussian distribution, the measured energy spread, $\delta_{\mathrm{M}}$ differs from the true SES, $\delta$, by

$$
\delta_{\mathrm{M}}=\sqrt{\delta^{2}+\delta_{\mathrm{res}}^{2}+\delta_{\mathrm{PW}}^{2}},
$$

with XTCAV energy resolution $\delta_{\text {res }} \approx 300 \mathrm{keV}$. Because $\delta_{\mathrm{PW}}$ increases linearly with XTCAV voltage, we operate at the minimum XTCAV voltage at which slice characteristics are observable. Figure 15 gives an example of a $0.5 \mathrm{kA}$ beam with XTCAV voltage set to $V=8 \mathrm{MV}$.

To determine the PW effect experimentally, we measure the SES as a function of XTCAV voltage, as shown in Fig. 16. We repeat the measurement at each peak current

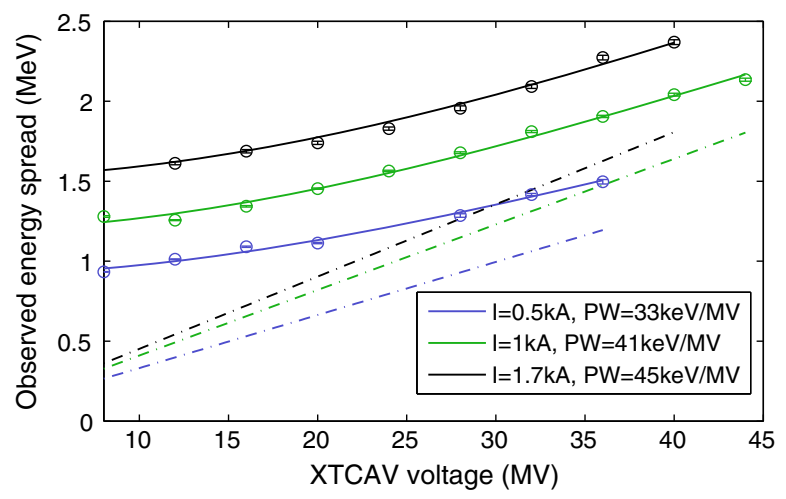

FIG. 16. Measured slice energy spread as a function of XTCAV voltage. The solid line is a fit of the form given in Eq. (E2), and the dotted line shows the contribution to the fit from the XTCAV (33-45 keV/MV, with 2-4\% fit uncertainty). The stronger PW effect at higher currents is due to larger beam size, either from a worse match or larger slice emittance. Data taken with $22 \mathrm{keV}$ energy spread induced by the laser heater.

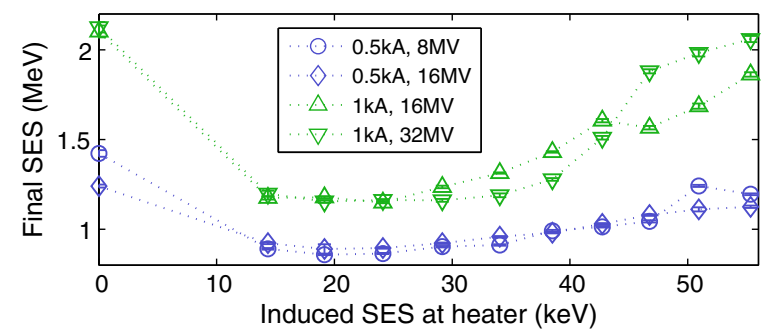

FIG. 17. SES measurements are consistent at different XTCAV voltages, confirming accuracy of the PW measurement, which is subtracted in quadrature.

setting because PW amplitude depends on beam size, which changes with current-dependent parameters such as emittance and $\beta$-function match. The result is consistent with the predicted values; PW contributes 33-45 $\mathrm{keV} / \mathrm{MV}$, with the larger values at higher peak current. We can also compare the measured PW effect to the estimate from Eq. (E1); the typical emittance is about $0.5 \mu \mathrm{m}$, and the designed horizontal beta function at XTCAV is $372 \mathrm{~m}$ at $4.3 \mathrm{GeV}$. Plugging these beam parameters into Eq. (E1) gives $36 \mathrm{keV} / \mathrm{MV}$, which is in reasonable agreement with the measurement.

For the measurements in Fig. 9, we set the XTCAV voltage so that the PW effect accounts for less than $20 \%$ of the measured energy spread, and as little as 5\% for the $0.5 \mathrm{kA}$ case. We can also check the validity of the PW contribution by repeating the SES measurements with higher XTCAV voltage (which gives better slice resolution, but also larger PW). Figure 17 shows reasonable agreement after subtracting off the PW contribution for two different XTCAV settings. Figures 9(b)-(c) are taken with even larger XTCAV voltage when PW dominates the measured SES. To match the data to the SES level of Fig. 9(a) (red points) we only need a 7-10\% tweak to the measured PW strength, which is within the uncertainty of the PW measurement, confirming validity of the PW values. (Note that Figs. 9(b)-(c) are concerned only with the trend in the SES amplitude, which is not affected by errors due to $\mathrm{PW}$.)

\section{APPENDIX F: SLICE ENERGY SPREAD METRICS}

To find the beam's SES, we measure the energy distribution of each pixel lineout of the XTCAV camera. From the lineout, we use two different metrics to quantify SES. First, we calculate the fwhm empirically and, assuming a Gaussian distribution, approximate $\mathrm{rms}=\mathrm{fwhm} /$ 2.35; though the distribution is not precisely Gaussian, the deviation is small. Second, after identifying the centroid, we can directly calculate the second moment independently for each side of the distribution. Figure 18 gives an example of both metrics applied to a single lineout. 


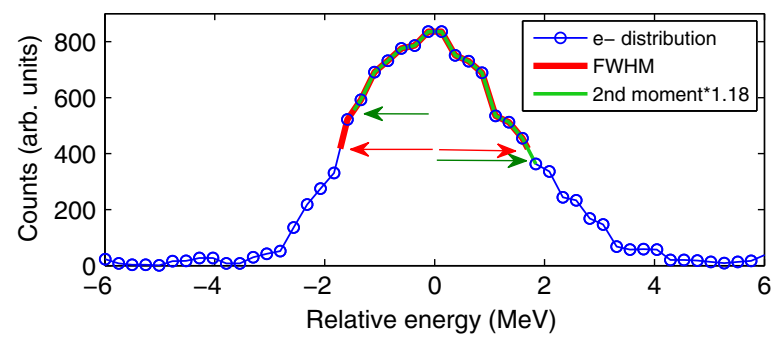

(a)

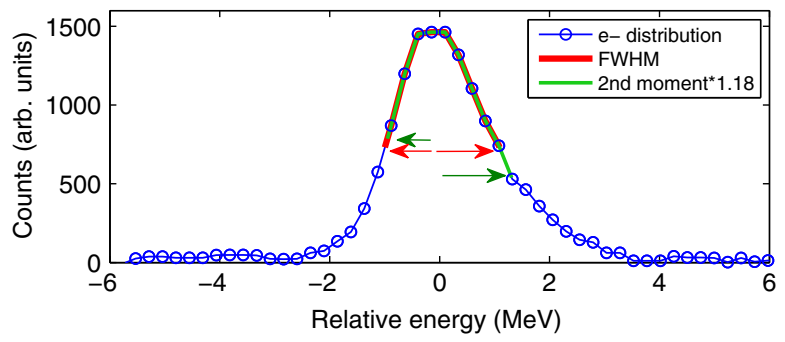

(b)

FIG. 18. Method used to determine the SES with the XTCAV. Examples given for a $0.5 \mathrm{kA}$ beam with laser heater set to induce energy spread of $8 \mathrm{keV}$ (a) and $30 \mathrm{keV}$ (b). An individual lineout (blue line with circles) is taken from the XTCAV image (e.g., Fig. 15), and either the fwhm (red lines/arrows) or the second moment (green lines/arrows) are determined from the lineout. The second moment is calculated individually for the left- and right-hand sides of the distribution, and then scaled by a factor of 1.18 to match the fwhm, assuming a Gaussian distribution. On the right side, the low shoulder biases the rms metric to give a larger energy spread.

Figure 19 shows SES as a function of longitudinal position along the beam for 30 shots with both the fwhm and second moment metrics. The results in the main paper (Fig. 9) use an average of the SES across just the core of the beam, where the SES is relatively constant. The high current "horns" at the ends have significantly higher energy

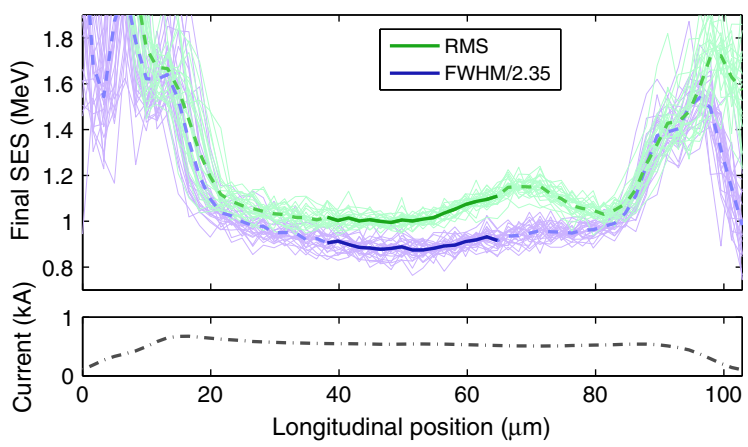

FIG. 19. Slice energy spread as a function of beam position for both the rms (green) and fwhm (blue) metrics. Light colored lines are for individual shots, and bold dashed lines show the average. Energy spreads in Fig. 9 are calculated by averaging over the portion with bold, solid lines. Average current profile of the beam shown below. spread due to a combination of wakefield effects and compression factor. The rms metric consistently gives larger SES values in the core of the beam, because of the high-energy shoulder evident in Fig. 18. There is also a notable bump in the rms method around $70 \mu \mathrm{m}$, where the shoulder increases in size.

The second moment has the advantage of being a rigorous metric without any assumptions about the distribution shape, however it has the disadvantage of sensitivity to assumptions about noise and background levels. Moreover, shoulders on the distribution can bias the second moment, even though these electrons may not participate in the FEL process. The fwhm metric emphasizes the core of the bunch, so we use this metric throughout.

\section{APPENDIX G: LASER HEATER CALIBRATION}

To convert the laser heater power to an induced energy spread on the electron beam, we measure the slice energy spread with the transverse deflecting cavity TCAV0 located after the laser heater. Just as for the XTCAV, the PW effect (Appendix E) increases the measured slice energy spread. However, except at the lowest laser heater settings, the PW effect from TCAV0 is negligible. Figure 20 shows the measured energy spread, $\delta_{\mathrm{M} 0}$, as a function of laser heater pulse energy, $p$. The solid blue line shows a fit of the form $[7,20]$

$$
\delta_{\mathrm{M} 0}(p)=\sqrt{\delta_{0}^{2}+\delta_{\mathrm{PW} 0}^{2}+\delta_{\mathrm{res} 0}^{2}+A p}
$$

with initial energy spread $\delta_{0}$, PW effect from TCAV0 of $\delta_{\text {PW0 }}$, TCAV0 resolution $\delta_{\text {res0 }}^{2}$, and fitting parameter $A$, which depends on the laser heater alignment and setup. The dashed red line in Fig. 20 shows the portion of the measured energy spread due to the heater. The deviation between the inferred laser heater contribution (red line) and measurement (blue line) is due to a combination of the

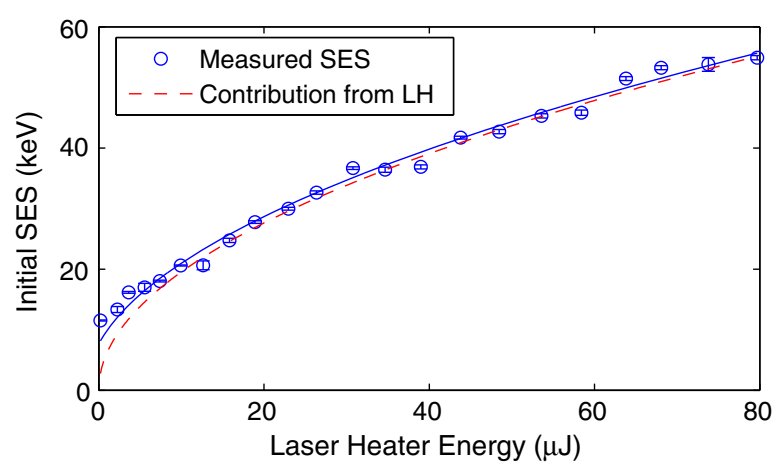

FIG. 20. Initial SES following the laser heater as a function of laser heater energy. Line shows a fit to Eq. (G1). Dashed red line shows the SES induced by the laser heater (LH), inferred from the fit. The deviation is due to the beam's initial energy spread from the gun, the PW effect, and the TCAV0 resolution. 
initial energy spread from the cathode and resolution limits, given by $\sqrt{\delta_{0}^{2}+\delta_{\mathrm{PW} 0}^{2}+\delta_{\text {res } 0}^{2}} \sim 10 \mathrm{keV}$.

Throughout the paper, we refer to the laser heater's initial contribution to SES, $A p$, as the "induced SES." We choose $A p$, rather than the full measured $\delta_{\mathrm{M} 0}$, as the metric, to remove the measurement artifacts of $\mathrm{PW}$ and resolution, which can dominate when the heater is weak. Using the induced SES for our metric ignores the contribution from the energy spread of the beam in absence of the laser heater, estimated from previous studies to be $\delta_{0} \sim 3 \mathrm{keV}[10,42]$, but this is a small quantity compared to the laser heater induced spread.

It is interesting to note that Fig. 20 shows no evidence of the "trickle heating" effect reported in [20]. Trickle heating is the generation of anomalously large energy spread when the laser heater itself seeds a transverse density modulation that drives MBI in later stages. Trickle heating is therefore sensitive to the beam optics in the accelerator following the heater chicane, and the optics parameters were not conducive to trickle heating during the period of data acquisition.

\section{APPENDIX H: ANALYTICAL MODEL OF ENERGY-SPREAD GROWTH}

The SES growth induced by the microbunching instability can be modeled with the space-charge induced microbunching theory from Huang et al. [7]. The SES at the beam-dump $(\delta)$ is a combination of the initial energyspread (from the cathode, $\delta_{0}$, and the laser heater, $A p$ ) multiplied by the compression factor $(C)$ summed in quadrature with the energy-spread induced by collective effects $\left(\delta_{c}\right)$,

$$
\delta=\sqrt{C^{2}\left(\delta_{0}^{2}+A^{2} p^{2}\right)+\delta_{c}^{2}} .
$$

We assume that the largest contribution to $\delta_{c}$ comes from the space-charge impedance after $\mathrm{BC} 2$, when peak current and existing microbunching is strongest. In this model, we ignore all SES growth before L3, which is a good assumption for the regime $A p \gtrsim 15 \mathrm{keV}$, when the SES before $\mathrm{BC} 2$ is dominated by the laser heater.

The SES from collective effects can be calculated by integrating the energy modulation induced by space-charge over all frequency components

$$
\delta_{c}^{2}=\left(m c^{2}\right)^{2} \frac{1}{2 \pi n_{z}} \int_{-\infty}^{+\infty} d k\left|G(k)^{2} \Delta \gamma(k)^{2}\right|,
$$

where $G(k)$ is the microbunching gain up to $\mathrm{BC} 2$ and $n_{z}$ is the linear density of the electron bunch (number of particles per unit length). The energy modulation per unit bunching factor from L3 (no compression) is given by [7]
$\Delta \gamma(k)=-\frac{I}{I_{A}} \int d s \frac{4 \pi Z_{\mathrm{lsc}}(k, z)}{Z_{0}}$,

with longitudinal space-charge impedance

$$
Z_{\mathrm{lsc}}=\frac{i Z_{0}}{\pi k r_{b}^{2}}\left[1-\frac{k r_{b}}{\gamma} K_{1}\left(\frac{k r_{b}}{\gamma}\right)\right]
$$

and where we have assumed a cylindrical beam distribution of radius $r_{b} . K_{1}$ is the first-order modified Bessel function of the second kind. The model results in negligible gain from the laser heater chicane and dog-legs DL1 and DL2, so the gain function is calculated only from $\mathrm{BC} 1$ and $\mathrm{BC} 2$.

The SES induced by the laser heater in the gain formula assumes the laser heater radius is double that of the electron beam [7]. The initial energy spread existing before the laser heater is modeled with a Gaussian distribution.

Integrating Eq. (H2) using the measured LCLS parameter we can estimate the final SES as a function of the laserheater induced spread. Figure 21 shows the computed SES as a function of the heater-induced spread for the $1 \mathrm{kA}$ case. For comparison the measured curve is also shown. Since the initial Gaussian spread is not known exactly (although it has been estimated to be in the few keV range [10]), the plot shows the computed SES for two values of the initial Gaussian component: $1 \mathrm{keV}$ (red line) and $3 \mathrm{keV}$ (blue line). The discrepancy between the $1 \mathrm{keV}$ and $3 \mathrm{keV}$ cases, even with $20 \mathrm{keV}$ spread induced by the laser heater, highlights the inefficient suppression of MBI with a mismatched laser heater distribution. The theoretical curve qualitatively reproduces the measured shape, with a minimum value between the MBI-dominated region and the laser-heater dominated region. Note that, since the microbunching theory assumes small microbunching (linear gain approximation) and ignores SES growth prior to L3, it overestimates SES for low heater power. The minimum value of the energy spread is in rough agreement with the theory which predicts SES between $0.65 \mathrm{MeV}$ and $1 \mathrm{MeV}$.

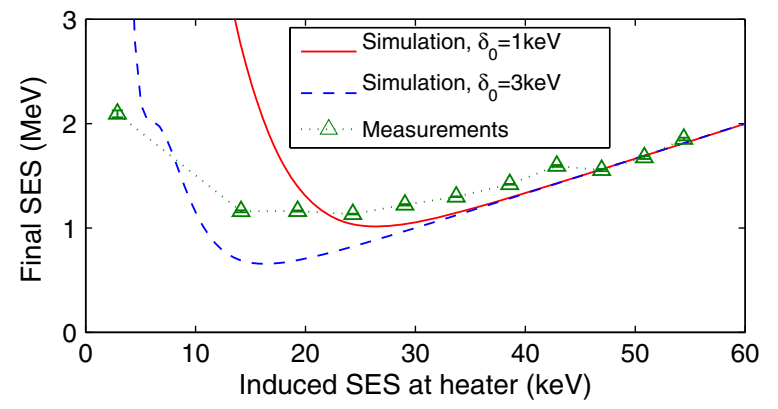

FIG. 21. Final energy spread as a function of laser-heater induced energy spread for the $1 \mathrm{kA}$ working point. The analytical calculation is shown for a Gaussian spread (before the heater) of $1 \mathrm{keV}$ (red, solid) and $3 \mathrm{keV}$ (blue, dashed). The green triangles show the XTCAV measurement. 
[1] M. Borland et al., Nucl. Instrum. Methods Phys. Res., Sect. A 483, 268 (2002).

[2] E. L. Saldin, E. A. Schneidmiller, and M. V. Yurkov, Nucl. Instrum. Methods Phys. Res., Sect. A 483, 516 (2002).

[3] E. L. Saldin, E. A. Schneidmiller, and M. V. Yurkov, Nucl. Instrum. Methods Phys. Res., Sect. A 490, 1 (2002).

[4] S. Heifets, G. Stupakov, and S. Krinsky, Phys. Rev. ST Accel. Beams 5, 064401 (2002).

[5] Z. Huang and K. J. Kim, Phys. Rev. ST Accel. Beams 5, 074401 (2002).

[6] E. Saldin, E. Schneidmiller, and M. Yurkov, Nucl. Instrum. Methods Phys. Res., Sect. A 528, 355 (2004).

[7] Z. Huang, M. Borland, P. Emma, J. Wu, C. Limborg, G. Stupakov, and J. Welch, Phys. Rev. ST Accel. Beams 7, 074401 (2004).

[8] T. Shaftan and Z. Huang, Phys. Rev. ST Accel. Beams 7, 080702 (2004).

[9] M. Venturini, Phys. Rev. ST Accel. Beams 10, 104401 (2007).

[10] D. Ratner, A. Chao, and Z. Huang, in Proceedings of the 2008 FEL Conference, Gyeongju, Korea (2008), p. TUPPH041.

[11] D. Ratner, Ph.D. thesis, Stanford University, 2011.

[12] A. Marinelli and J. B. Rosenzweig, Phys. Rev. ST Accel. Beams 13, 110703 (2010).

[13] A. Marinelli, E. Hemsing, and J. B. Rosenzweig, Phys. Plasmas 18, 103105 (2011).

[14] R. Akre et al., Phys. Rev. ST Accel. Beams 11, 030703 (2008).

[15] H. Loos et al., in Proceedings of the 2008 FEL Conference, Gyeongju, Korea (2008), p. THBAU01.

[16] B. Schmidt, C. Behrens, H. Delsim-Hashemi, P. Schmuser, and S. Wesch, in Proceedings of the 11th European Particle Accelerator Conference, Genoa, 2008 (EPS-AG, Genoa, Italy, 2008).

[17] A. H. Lumpkin, R. J. Dejus, and N. S. Sereno, Phys. Rev. ST Accel. Beams 12, 040704 (2009).

[18] A. H. Lumpkin, N. S. Sereno, W. J. Berg, M. Borland, Y. Li, and S. J. Pasky, Phys. Rev. ST Accel. Beams 12, 080702 (2009).

[19] S. Wesch, C. Behrens, and B. Schmidt, in Proceedings of the 2009 FEL Conference, paper WEPC50 (Liverpool, UK, 2009).

[20] Z. Huang et al., Phys. Rev. ST Accel. Beams 13, 020703 (2010).
[21] H. Tanaka, in Proceedings of the 2nd International Particle Accelerator Conference, San Sebastiáán, Spain (EPS-AG, Spain, 2011).

[22] C. Behrens, C. Gerth, G. Kube, B. Schmidt, S. Wesch, and M. Yan, Phys. Rev. ST Accel. Beams 15, 062801 (2012).

[23] S. Spampinati et al., Phys. Rev. ST Accel. Beams 17, 120705 (2014).

[24] J. Galayda, in Proceedings of IPAC2014 (Dresden, Germany, 2014), p. TUOCA01.

[25] C. Behrens et al., Nat. Commun. 5, 3762 (2014).

[26] L. H. Yu, Phys. Rev. A 44, 5178 (1991).

[27] E. A. Schneidmiller and M. V. Yurkov, Phys. Rev. ST Accel. Beams 15, 080702 (2012).

[28] D. Ratner et al., in Proceedings of the 2013 FEL Conference (New York, New York, 2013), p. 15743.

[29] E. A. Schneidmiller and M. V. Yurkov, Phys. Rev. ST Accel. Beams 13, 110701 (2010).

[30] A. Marinelli, E. Hemsing, M. Dunning, D. Xiang, S. Weathersby, F. O'Shea, I. Gadjev, C. Hast, and J. B. Rosenzweig, Phys. Rev. Lett. 110, 264802 (2013).

[31] D. Ratner, Z. Huang, and G. Stupakov, Phys. Rev. ST Accel. Beams 14, 060710 (2011).

[32] D. Ratner and G. Stupakov, Phys. Rev. Lett. 109, 034801 (2012).

[33] S. Seletskiy, Y. Hidaka, J. B. Murphy, B. Podobedov, H. Qian, Y. Shen, X. J. Wang, and X. Yang, Phys. Rev. ST Accel. Beams 14, 110701 (2011).

[34] Z. Huang, J. Wu, and T. Shaftan, ICFA Beam Dynamics Newsletter, No. 38 (2005), http://icfa-usa.jlab.org/archive/ newsletter/icfa_bd_nl_38.pdf.

[35] D. Ratner et al., in Proceedings of the 2009 FEL Conference, Liverpool, England (2009), p. TUOA03.

[36] S. Di Mitri, M. Cornacchia, S. Spampinati, and S. Milton, Phys. Rev. ST Accel. Beams 13, 010702 (2010).

[37] S. Di Mitri and S. Spampinati, Phys. Rev. Lett. 112, 134802 (2014).

[38] W. K. H. Panofsky and W. Wenzel, Rev. Sci. Instrum. 27, 967 (1956).

[39] C. Behrens, Z. Huang, and D. Xiang, Phys. Rev. ST Accel. Beams 15, 022802 (2012).

[40] D. Ratner et al., Phys. Rev. Lett. 114, 054801 (2015).

[41] G. Penco, E. Allaria, G. De Ninno, E. Ferrari, and L. Giannessi, Phys. Rev. Lett. 114, 013901 (2015).

[42] M. Hüning and H. Schlarb, in Proceedings of the 20th Particle Accelerator Conference, PAC-2003, Portland, OR, 2003 (IEEE, New York, 2003). 